\title{
An account of spin memristive and memcapacitive systems: Next generation memory devices
}

\author{
Raj Kumar Singh ${ }^{1,2 *}$ and Kumari Mamta ${ }^{3,4}$ \\ ${ }^{1}$ Department of Electronics and Telecommunications, Politecnico di Torino, Torino 10129, Italy \\ ${ }^{2}$ Department of Physics, R. L. S. Y. College, Ranchi University, Ranchi, Ranchi 834001, India \\ ${ }^{3}$ Dept. of Electronic Engineering and Telecommunications, Politecnico di Torino, Torino 10129, Italy \\ ${ }^{4}$ Department of Physics, Jharkhand Rai University, Ranchi 834002, India
}

\begin{abstract}
We explore the current state of the progress in memristive and memcapacitive devices and their possible applications in electronic, spintronic and in the field of biological neural network from the first proposition by Leon O. Chua in 1971. This demands inclusion of devices exhibiting memristive behavior due to change in internal state following variations in temperature, spin polarization, resistance and history dependent channel conductance in axons. Memcapacitive systems that can be realized from metamaterial medium with metal layer embedding, from charge transport through nanopore, and by the vibration of strained elastic membrane replacing one plate of the regular capacitor has also been discussed. Finally, memristor and memcapacitor based circuits finding application in analog memory and computing have been reported.
\end{abstract}

Keywords: memristor, memcapacitor, non-pinched hysteresis, pinched hysteresis, spin memory.

\section{Introduction}

Memory means recalling past experiences in human being and in the field of information technology, it is the ability to store and retrieve digital information for use in computation. Interestingly, memory state of a system is related to some of the dynamical properties of electrons and ions, indeed, related to their rearranging behavior under the effect of external perturbations. Further, such a change of state of electrons and ions is not instantaneous, and it has something to do with their past dynamics [1]. This means that the resistive, capacitive and/or inductive properties of (nanoscale) systems depend on the past states through which the system has evolved.

Recent discovery of the memristor has paved way to new directions in optimization and revolutionizing analog circuit design, marking a new era for the advancement of analogue applications. In 1971, Leon Chua [3] postulated the existence of the fourth 'missing' two-terminal passive fundamental circuit element, called the memristor, short for memory-resistor. This device, as observed by Leon Chua, provides a functional relationship between the time integrals of voltage and current. The uniqueness of memristor is its ability to remember its history by way of time evolution of some internal state variable $x$ of the device. As the present day CMOS technology is approaching the nano-scale floor overriding the Moore's law, the dimensionality, the power management and the dynamic nonlinear response of memristive devices qualifies them as a hot research entity.

Outline of the paper is as follows: The paper is divided into three parts: Part A, Part B and Part C. In Part A, section A1 introduces the concept and properties of memory circuit elements with special reference to memristor and memristance. In section A 2, we pick up the case of various systems exhibiting memristive behavior like thermistor, HP memristor, semiconductor/half metal junction and biological neural network to discuss how memristive behavior comes to them naturally. In section A 3, we discuss various possible applications of memristors in realizing electronic, spintronic, neural network devices. Part B is devoted to memcapacitor and memcapacitive systems. Section B 1 introduces the concept and properties of memcapacitor. In section B 2, we discuss about the various systems exhibiting memcapacitive behavior like a solid-state memcapacitor realized from a metamaterial medium consisting of $N$ metal layers embedded into an insulator, inserted between the plates of a regular capacitor, ionic-memcapacitor based on the transport of charge through a nanopore and a mechanical-memcapacitor realized from the vibration of a strained elastic membrane replacing one of the plates of a regular parallel plate capacitor. Concluding remarks can be found in part C.

\section{A. MEMRISTOR AND MEMRISTIVE SYSTEMS \\ A1. General definition: memory circuit elements}


We know from classical circuit theory that there are three fundamental circuit elements ( $\mathrm{R}, \mathrm{C}$ and $\mathrm{L})$ associated with four basic circuit (charge, voltage, current and flux). For linear elements these relations take the following forms:

$$
V=R I
$$

for a resistor of resistance $\mathrm{R}$, given the current $\mathrm{I}$ and the voltage response $\mathrm{V}$.

$$
q=C V_{C}
$$

for a capacitor of capacitance $C$ that holds a charge $q$ and sustains a voltage $V_{C}$ and

$$
\phi=L I
$$

When the flux $\phi$ is generated by an inductor of inductance $L$ when a current I flows across it.

In the equations (1-3), the constants $\mathrm{R}, \mathrm{C}$ and $\mathrm{L}$ describe the linear response of the resistor, capacitor and inductor and hence are the response function.

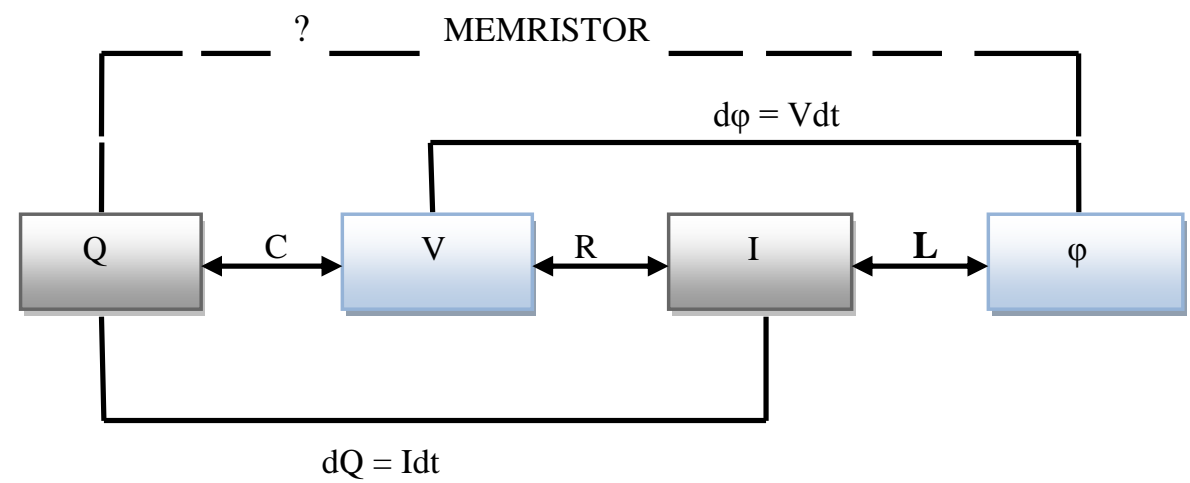

Fig.1. Four fundamental circuit elements: Resistance $(\mathrm{V}=\mathrm{RI})$, capacitance $(\mathrm{Q}=\mathrm{CV})$, inductance $(\varphi=\mathrm{LI})$, and memristance $(\varphi=\mathrm{Mq})$ which is the missing link as suggested by Chua.

Leon Chua was the first to find the missing link between the flux and charge in 1976 (see fig. 1and fig. 3 for typical flux Vs charge relation).

We can generalize the above definition to time dependent and non-linear responses as well, which may have memory dependence on other state variables also. If $u(t)$ and $y(t)$ are any two complementary constitutive circuit variables (current, charge, voltage, or flux) denoting input and output of the system, respectively, and $x$ is an ndimensional vector of internal state variables, we may then postulate the existence of the following nth-order $u$ controlled memory element as that defined by the equations [2].

$$
\begin{aligned}
& y(t)=g(x, u, t) u(t) \\
& \dot{x}=f(x, u, t)
\end{aligned}
$$

Here, $g$ is a generalized response, and $f$ is a continuous n-dimensional vector function.

A distinctive signature of memory devices is a hysteresis loop which follows when the response function $g(t)$ or the function $y(t)$ (or both) are plotted versus $u(t)$. The shape of a loop is determined by both the device properties and the input $u(t)$ applied. For well-defined generalized response functions $g$, the function $y(t)$ hysteresis loop passes through the origin ( $y$ is zero whenever $u$ is zero and vice versa). This is called a "pinched" hysteresis loop. A pinched loop may be "self-crossing- type I crossing behavior " or "not self-crossing- type II crossing behavior"(see fig. 2). 

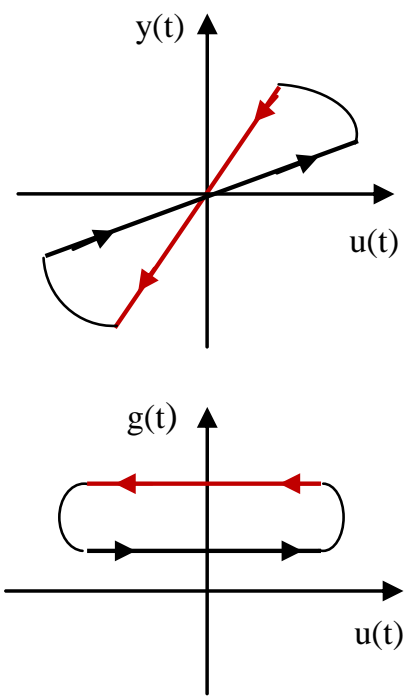

Type I
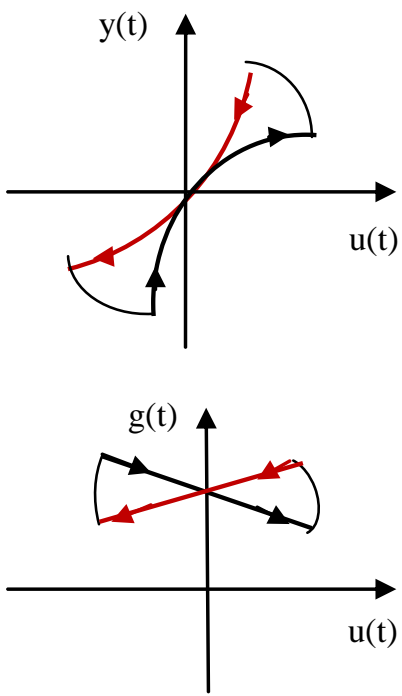

Type II

Fig. 2 Schematic pinched hysteresis loop of memory elements when subject to a periodic stimulus can be "self-crossing" (type I) or not (type II). The latter property often (but not always) arises when the state dynamics function $f$ and the response function $g$ are even functions of the input variable $u$.

\section{Memristors}

Memristor, the contraction of - memory resistor, is a passive device that provides a functional relation between charge and flux. It is defined as a two-terminal circuit element in which the flux between the two terminals is a function of the amount of electric charge that has passed through the device [9]. A memristor could be chargecontrolled or flux-controlled. It is said to be charge-controlled if the relation between flux and charge is expressed as a function of electric charge and is said to be flux-controlled if the relation between flux and charge is expressed as a function of the flux linkage [3].

For a charge-controlled memristor,

$$
\phi=f(q)
$$

Differentiating equation (6) yields

$$
\frac{d \phi}{d t}=\frac{d f(q)}{d q} \times \frac{d q}{d t}
$$

Or,

$$
v(t)=M(q) i(t)
$$

where,

$$
v(t)=d \phi / d t \text { is the voltage }
$$

and

$$
M(q)=d f(q) / d q
$$

$M(q)$ is called as memristance, and it has the units of resistance. It defines a linear relationship between current and voltage, as long as the charge does not vary. Thus if $M$ is constant, a memristor behaves as a resistor. Memristance is a property of the memristor. When the charge flows in one direction through a circuit, the resistance of the memristor increases, and its resistance decreases when the charge flows in the opposite direction in the circuit. If the applied voltage is turned off, thus stopping the flow of charge, the memristor "remembers" the last resistance that it had. When the flow of charge is started again, the resistance of the circuit will be what it was when it was last active. A useful analogy for a memristor is to think of its behaviour as being like the flow of water through a pipe whose diameter expands or shrinks depending on the direction and amount of water that has flowed through it. When the water flow is turned off, the pipe retains its current diameter, thus 'remembering' the amount of water that has passed. 
For a flux-controlled memristor,

$$
q=f(\phi)
$$

Differentiating equation (10) yields

$$
\frac{d q}{d t}=\frac{d f(\phi)}{d \phi} \times \frac{d \phi}{d t}
$$

or

$$
i(t)=W(\phi) v(t)
$$

where

$$
i(t)=d q / d t \text { is the current }
$$

$W(\phi)=d f(\phi) / d \phi$, called as memductance ( the reciprocal of memristance) and it has the unit of conductance.

For non-linear response system with memory dependence on some state variable $x(t)$ where the time evolution of $x$ is known to be

$$
d x / d t=f(x, I, t)
$$

where $f$ is a continuous n-dimensional vector function, the relation between voltage and current can then be written as

$$
V(t)=R_{M}(x, I, t) I(t)
$$

and needs to be solved together with equation (13) for the state variables dynamics. These systems (with $f$ and or $R_{M}$ depending on $I$ have been called current-controlled memristive systems [13]. The voltage-controlled ones are those that satisfy the relations

$$
\begin{gathered}
I(t)=G(x, V, t) V(t) \\
\dot{X}=f(x, V, t)
\end{gathered}
$$

where $G$ is called the memductance (for memory conductance).

Properties of memristors

Memristive devices are passive for all $\mathrm{R}>0$.

A memristive system can't store energy, like a capacitor or an inductor. This is quite obvious from the fact that $\mathrm{V}=0$ whenever $\mathrm{I}=0$, and vice versa.

The most important one is the appearance of a "pinched hysteretic loop" (see fig. 4) in the current-voltage characteristics of these systems when subject to a periodic input [13]. During each period of the input voltage the I - V curve is a simple loop passing through the origin, namely there may be at most two values of the current I for a given voltage $\mathrm{V}$, if we consider a voltage controlled device, or two values of the voltage $\mathrm{V}$ for a given current I, for a current-controlled system.
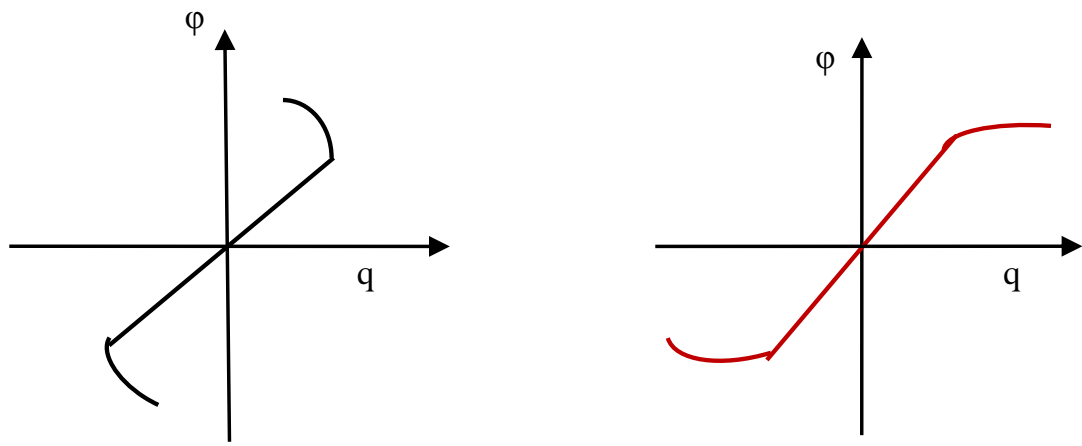

Fig. 3. Typical $\phi-\mathrm{q}$ curves of memristors 
A memristive system behaves as a linear resistor in the limit of infinite frequency and as a non-linear resistor in the limit of zero frequency. At very low frequencies, the system has enough time to adjust its value of resistance to a momentary value of the control parameter (either current or voltage), so that the device behaves as a nonlinear resistor. On the other hand, at very high frequency, there is not enough time for any kind of resistance change during a period of oscillations of the control parameter, so that the device operates as a usual (linear) resistor.

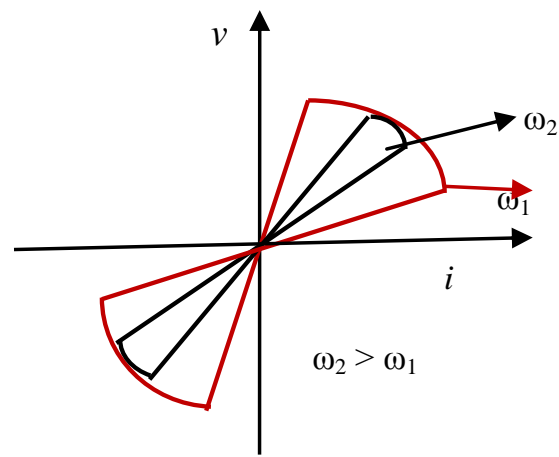

Fig. 4. The pinched hysteresis loop and the loop shrinking with the increase in frequency.

Memristive behavior has been observed in thermistors [7], molecular systems [8], spin electronic devices [9] and nanostructures due to thin films [10-12], as well as various examples mentioned in [13].

\section{A 2. Memristive systems}

\section{A 2.1. Thermistor}

One of the first identified memristive systems is the thermistor: a temperature sensitive resistor. Thermistors are built of semiconducting materials that are especially sensitive to temperature, such as different oxides of metals including manganese, iron, nickel, cobalt, copper, and zinc. Memristive properties of thermistors are based on self-heating and were noticed by Chua and Kang in 1976 [13].

Consider a thermistor with negative temperature coefficient that is described by the current-voltage relation [14]

$$
V=R_{0} e^{\beta\left(1 / T-1 / T_{0}\right)} I
$$

where the constant $R_{0}$ is the resistance at a temperature $T_{0}, T$ is the absolute temperature of thermistor and $\beta$ is a material-specific constant. Resistance of negative temperature coefficient thermistor decreases with temperature. The time evolution of thermistor temperature is given by the heat transfer equation

$$
C_{h} \frac{d T}{d t}=V(t) I(t)+\delta\left(T_{e n v}-T\right)
$$

where $C_{h}$ is the heat capacitance, $\delta$ is the dissipation constant of the thermistor [13], and $T_{e n v}$ is the ambient temperature. Temperature $T$ of the thermistor plays the role of the internal state variable.

\section{A 2.2. Hewlett-Packard (HP) memristor}

The Hewlett-Packard (HP) memristor was realised on the concept of drifting the dopant between doped and undoped portion of the material, which models the memristive property.

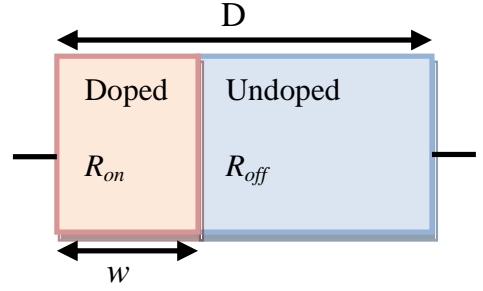

(a)

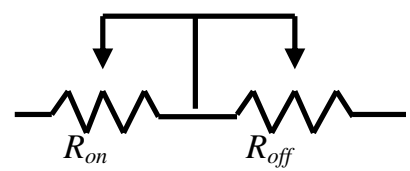

(b)

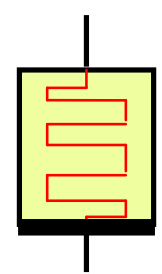

(c)

Fig. 5. (a) Memristor device structure. (b) Equivalent circuit model. (c) Memristor symbol [5]. 
Fig. 5(a), (b) and (c) show the physical structure of a memristor device along with its equivalent circuit model and symbol [4]. The device is an electrically switchable semiconductor thin film sandwiched between two metal contacts. There are two layers in the titanium dioxide film. The semiconductor thin film has a certain length ,

and consists of double layers of titanium dioxide films. One is highly resistive pure $\mathrm{TiO}_{2}$ (undoped layer), and the other is filled with oxygen vacancies, which makes it highly conductive (doped layer). The state variable $w$ represents the width of the doped region $\left(\mathrm{TiO}_{2-\mathrm{x}}\right.$ layer). The doped region has low resistance while that of the undoped region is much higher. The boundary between the doped and undoped regions, and therefore the effective resistance of the thin film, depends on the position of +2 mobile dopants. It, in turn, is determined by their mobility $\left(\sim 10^{-10} \mathrm{~cm}^{2} \mathrm{~V}^{-1} \mathrm{~s}^{-1}\right)$ [9] and the electric field across the doped region.
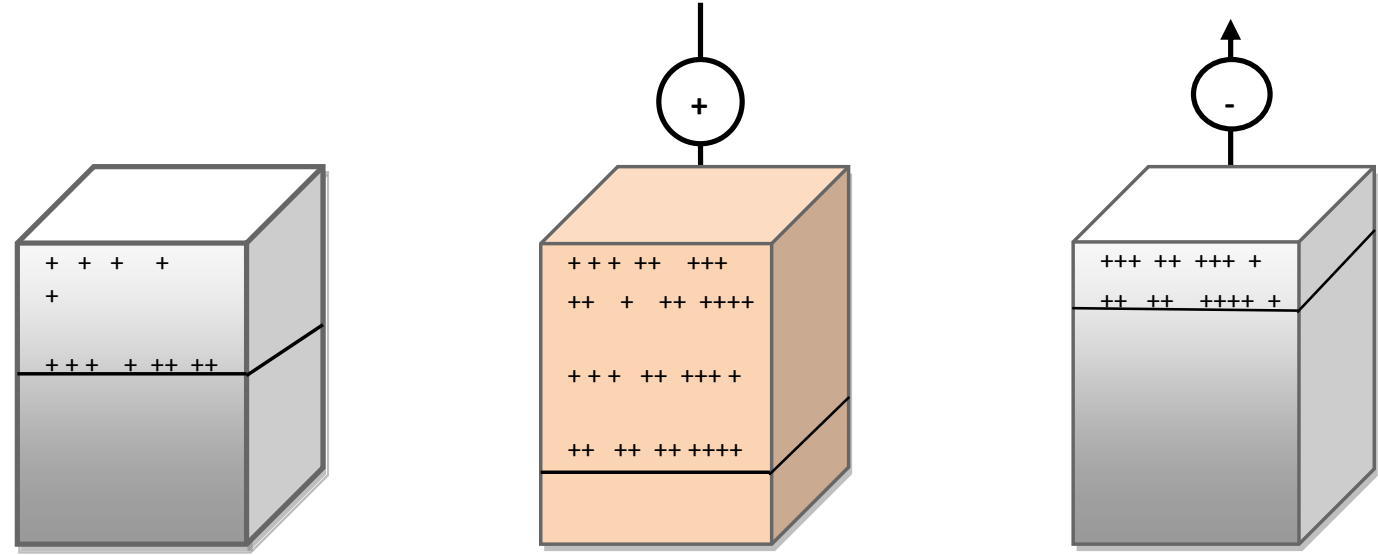

Fig.6. Change in $w$, the width of doped region, of HP memristor when the external bias $v$ is applied across it. + symbol represents oxygen deficiencies.

As an external voltage bias $v$ is applied across the device, the electric field will repel positively charged oxygen vacancies in the doped layer into the pure $\mathrm{TiO}_{2}$ layer resulting the length $w$ changed [11] (see fig. 6). Hence, the device's total resistivity changes. If the doped region extends to the full length $D(w=D)$, then $w / D=1$, the total resistivity of the device would be dominated by the low resistivity region, with a value measured to be $R_{o n}$. Likewise, when the undoped region extends to the full length $D(w=0)$, i.e. $w / D=0$, the total resistance is denoted as $R_{\text {off }}$. According to [3], the memristor has memory effect since the device maintains its resistivity even if the power goes off. According to the reported device characteristics $[3,13]$, oxygen vacancies do not move around by themselves. They become absolutely immobile until voltage is applied again. This unique characteristic makes the memristor standout from other devices such as diode.

The mathematical model for memristive device resistance can be described as

$$
R(w)=R_{o f f}-\left(R_{o f f}-R_{o n}\right) \times w / D, \quad \text { where } 0 \leq w \leq D
$$

$R_{\text {on }}(\sim 1 \mathrm{k} \Omega)[12]$ corresponds to memristor state $w=D . R_{\text {off }}$ corresponds to memristor state $w=0$. The device resistance is bounded between $R_{o n} \leq R(w) \leq R_{o f f}$

\section{A 2.3. Semiconductor/half-metal junction memristor}

The use of the electron spin degree of freedom allows for the realization of memristive behavior. This fact is exploited through semiconductor/half-metal junction. Half metals are the ferromagnet with 100\% spin polarization at the Fermi level and act as perfect spin filters. The principal role in realizing the memory effect is played by electron-spin diffusion and relaxation processes which drives the system to equilibrium.

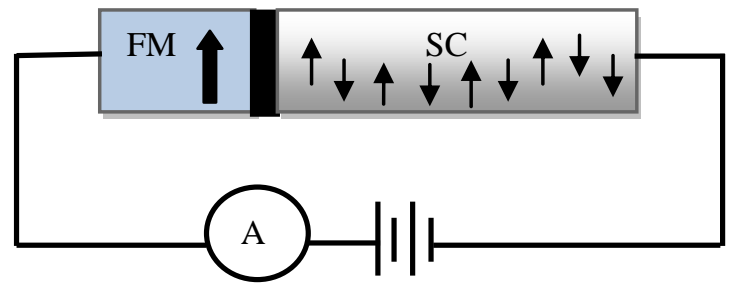

a 


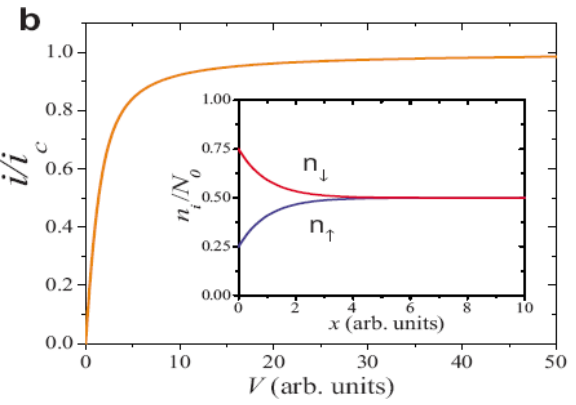

Fig 7.Semiconductor/half-metal junction. (a) Schematic representation of the circuit made of an interface between a semiconductor and a half metal. (b)Typical dc current-voltage characteristics. Inset: spin-up and spin-down densities in the semiconductor region as a function of the distance from the contact [15].

In a work by Yu. V. Pershin and M. Di Ventra [15] it has been shown that the spin blockade at such junctions leads to a saturated $i$ - $V$ curve (Fig. $7 b$ ) that such a system has all the necessary components to exhibit memristive behavior. The physics of the spin blockade lies in the perfect spin filter property of half metal: the half metal accepts electrons of only one-say up - spin direction. Spin-down electrons cannot enter the half metal and, therefore, form a cloud near the contact when a current flows through the system. This cloud increases with increasing current. At a critical current density the density of spin-up electrons near the contact becomes insufficient to provide a further current increase. In other words, transport of spin-up electrons through the contact is blocked by the cloud of spin-down electrons near the contact. It was predicted by Yu. V. Pershin and M. Di Ventra [16] that the spin blockade leads to a saturated $i$ - $V$ curve (as that shown in Fig. 7b). Fig. 8 is the transverse voltage as a function of applied electric field at different applied field frequencies.
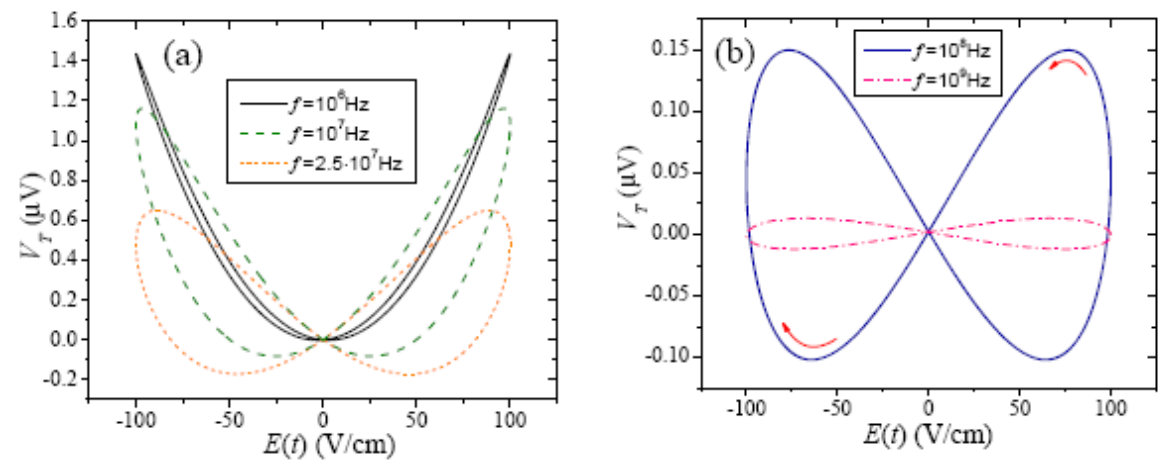

Fig 8. Spin memristive effects in a semiconducting system with inhomogeneous electron density in the direction perpendicular to main current flow [2]. Shown in this figure is the transverse voltage as a function of applied electric field at different applied field frequencies as indicated. From [2].

\section{A 2.4. Biological neural network memristor}

Another important memristive system pertains to biological neural networks-functioning of membranes in axon cells. In fact, in 1952 Hodgkin and Huxley suggested a model of action potentials [18] in neurons that employs history-dependent channel conductances that are essentially memristive. This model is one of the most significant conceptual achievements in neuroscience [17]. The nerve membrane was described by three types of ion channels:

leakage channels (primarily carrying chloride ions), Na channels and K channels.

Leakage channels have a relatively low constant conductance and are the source to the resting membrane potential. The conductance of $\mathrm{Na}$ channels and $\mathrm{K}$ channels depends and changes as a function of time and voltage, and herein lies the memristive behavior. Hodgkin and Huxley have demonstrated that the step depolarization initiates a rapid inward current across the membrane carried by $\mathrm{Na}+$ ions, followed by an outward current due to $\mathrm{K}+$ ions. For quantitative understanding, they have suggested an equivalent circuit model of the membrane. (see fig. 9). Mathematically, the membrane current is written as [18]. 


$$
\left.I=\frac{C_{m} d V_{m}}{d t}+M_{N a}^{-1}\left(V_{M}-E_{N a}\right)+M_{K}^{-1}\left(V_{M}-E_{K}\right)+R_{l}^{-1}\right)\left(V_{M}-E_{l}\right)
$$

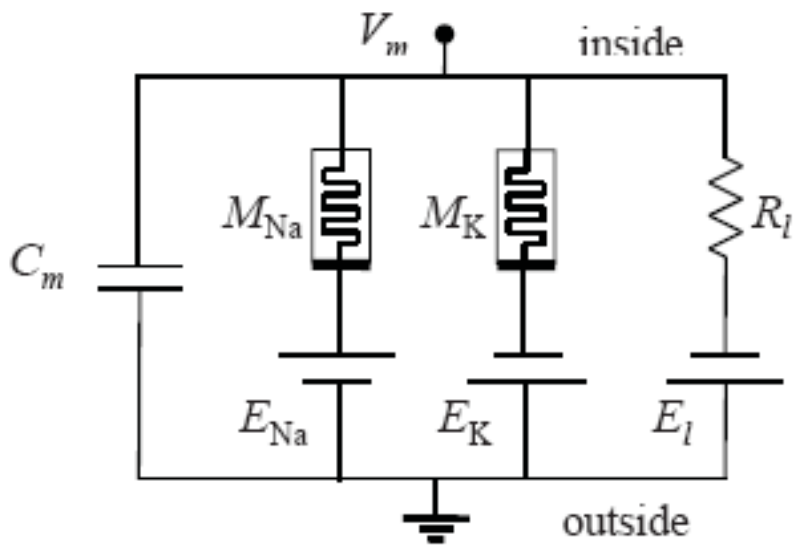

Fig .9: Equivalent electrical circuit for a short segment of squid axon membrane (modified from reference [18]. Here, $C_{m}$ is the membrane capacitance, $V_{m}$ is the membrane potential, $M_{N a}$ and $M_{K}$ are memristive systems describing conductivity of $\mathrm{Na}$ and $\mathrm{K}$ channels, respectively, $R_{l}$ is the leakage resistance, $E_{N a}, E_{K}$ and $E_{l}$ are reverse ion channel potentials, and the terms "inside" and "outside" reflect the interior of the axon.

where the memory conductances $M_{N a}^{-1}=g_{N a} m^{3} h, \quad M_{K}^{-1}=g_{K} n^{4}, M_{N a}^{-1}=g_{N a} \quad$ with $g_{N a}, g_{K}$, constants, $R_{l}$ describes the leakage resistance, and all other circuit quantities. The time-dependencies of voltage-dependent gating variables $n, m$ and $h$ are

$$
\begin{aligned}
& d n / d t=\alpha_{n}(1-n)-\beta_{n} n \\
& d m / d t=\alpha_{m}(1-m)-\beta_{m} m \\
& d h / d t=\alpha_{h}(1-h)-\beta_{h} h
\end{aligned}
$$

where $\alpha_{n}(m, h)$ and $\beta_{n}(m, h)$ are voltage-dependent constants [18] defined as

$$
\begin{aligned}
& \alpha_{n}=\frac{0.01\left(V_{m}+10\right)}{e^{\left(V_{m}+10\right) / 10}-1} \\
& \beta_{n}=0.125 e^{V_{m} / 80} \\
& \alpha_{m}=\frac{0.1\left(V_{m}+25\right)}{e^{\left(V_{m}+25\right) / 25}-1} \\
& \beta_{m}=4 e^{V_{m} / 18} \\
& \alpha_{h}=0.07 e^{V_{m} / 20} \\
& \beta_{h}=\frac{1}{e^{\left(V_{m}+80\right) / 10}+1}
\end{aligned}
$$

In the above equations, the voltage is in $m V$ and time is in $m s$. Time evolution of channels' conductance $n, m$ and $h$ values between 0 and 1. It follows from the expressions for $M_{N a}$ and $M_{K}$ (given in equation (17)) and equations (21-23) that the potassium and sodium channels can be classified as first-order and second-order voltage-controlled memristive systems [13]. In fig. 10 and 11 we present simulations of potassium and sodium channel memristive systems biased by ac-voltage due to Yuriy V. Pershin and Massimiliano Di Ventra [19]. These plots demonstrate frequency-dependent I- V curves typical of memristive systems. 

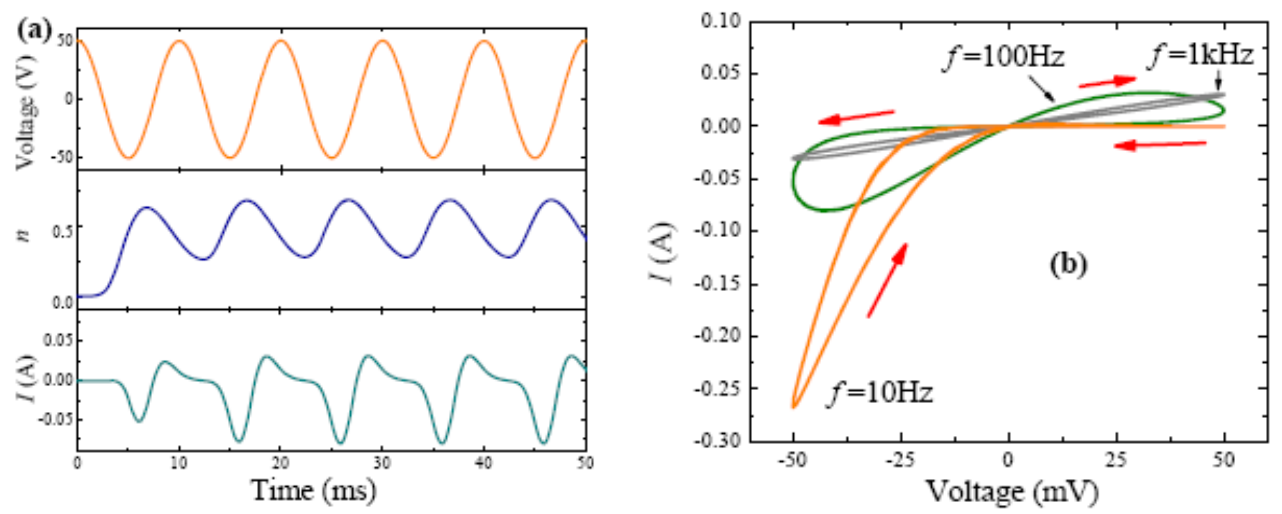

Fig 10. Simulations of the potassium channel memristive system $\left(M_{K}\right)$ response. We have used $g_{K}=10 \mathrm{mS}$. The applied voltage is $V(t)=V_{0} \cos (2 \pi f t)$ with $V_{0}=50 \mathrm{mV}$ and $f=100 \mathrm{~Hz}$ in (a). After [19].
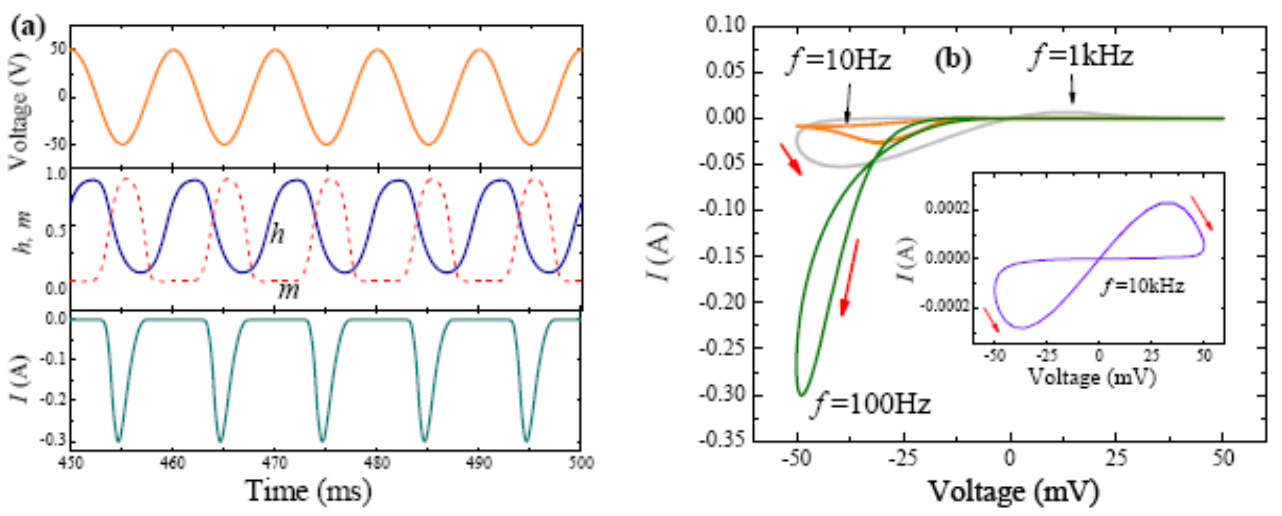

Fig 11. Simulations of the sodium channel memristive system $\left(M_{N a}\right)$ response. We have used $g_{N a}=33 \mathrm{mS}$. The applied voltage is $V(t)=V_{0} \cos (2 \pi f t)$ with $V_{0}=50 m V$ and $f=100 \mathrm{~Hz}$ in (a). After [19].

\section{A 3. Memristors application in memory and analog devices}

Associative memory

Yuriy V. Pershin and Massimiliano Di Ventra [20] have shown, through their memristor emulator, that an artificial synaptic function can be achieved by the use of memristor. It is worth mentioning here that synapses are at the core of computation and information storage and retrieval in neural systems. The emulator is capable of retaining past events/memories and storing a continuous set of states, at the same time it simulates to behave as good as 'plastic' as per the requirement of synaptic dynamics. Further, they have showed successfully that two memristor emulator synapses connected by simple neural network leads to the formation of associative memory, an important function of the brain. This way memristor finds application in reproducing complex learning and adaptive behavior, like that of human brain.

Read operation of memory

Affan Zidana et al, have demonstrated the read operation capability of memristors in one of their work [21]. The sneak paths problem has been analyzed with different memory array sizes, data sets, and architectures and following this a noise margin metric has been proposed successfully to compare the various available solutions. Memristor-based memory arrays has been used to assess the power consumption associated with the solutions and promising results have been obtained highlighting the important role played by memristors in solving sneak paths problems.

Analog memory

Mika Laiho and Eero Lehtonen [22] have highlighted the hidden potential of memristors in analog memory and computation through programming and comparison. Memristor has been programmed, at a fixed voltage, to achieve the desired conductance level. Further, the devices based on memristor can be reconfigured to perform 
the arithmetic operations viz. addition and subtraction of analog conductances of dual polarity. A model of memristor with nonlinear programming feature has been proposed that automatically stops the programming upon reaching the correct conductance value by way of a cyclical programming. Thus, arithmetic operations with memristor-based analog memory is going to become a reality very soon.

Analog devices

Several works on memristor have been reported discussing the realization of analog devices based on memristor.

Op-amp. Qin Yu et al [23] have studied the small signal transmission characteristics of a monotone-increasing and piecewise-linear memristor-based inverting and non inverting op-amp circuits and have realized the respective voltage gain.

Filter. Filter characteristics based on memristor has been reported by Wei Wang et al [24].

Adaptive filter. Memristive adaptive filters has been studied by T. Driscoll et al [25] in one of their works. They have demonstrated an adaptive ("learning") filter based on memristor. This filter responds to pre-specified signals by sharpening the quality factor of its response. Further, they claim to model the learning patterns observed in biological organisms through this adaptive filter. The analog functionality of memristive components is supposed to allow combination of signal processing in storage and fuzzy logic. The adaptive filter circuit consists only of passive basic circuit elements which is different from the other method of realizing adaptive filtering with combinations of transistors. Further, this device offers advantages in device density and power consumption.

Oscillator. Li Zhi-Jun and Zeng Yi-Cheng [26] have modeled, an inductance-free chaotic circuit based on a twin-T oscillator and memristor, by coupled first-order differential equations. The circuit evolves into different orbits, chaos, and hyperchaos upon change of control parameters.

To observe the chaotic behaviors in the circuit, they designed an analog realization of the piecewise-linear flux controlled memristor with conventional electronic devices. The circuit can find application in chaos-based communication and electronic measurement systems.

Phase shift oscillator. A. Talukdar et al [27], have reported the good potential application for parametric oscillation of the memristor in conventional phase shift oscillator. The effects of using the memristor in place of a conventional resistor have been described mathematically and are verified by the simulation results. The said systems qualifies as an autonomous parametric oscillatory system where the memristor sets its resistance as a periodically changing parameter.

The oscillating resistance, operating frequency range, and the dynamic poles are expected to redefine the conventional concept..

Relaxation oscillator. A. G. Mosad et al [28] have introduced a memristor-based relaxation oscillator free from any reactive components. They have obtained higher oscillation frequency over a wide range of resistance compared with that of existing reactance-less oscillators. The circuit can be operated with positive as well as and negative supplies meeting the oscillation condition. Authors strongly believe that the proposed oscillator provides a suitable solution for low frequency applications such as the biomedical and embedded systems applications.

Spintronic temperature sensor

Spintronic memristor based temperature sensor has been proposed by Xiaobin Wang et al [29] exploiting the rich dynamic behavior of memristor upon excitation with dynamic current/voltage profile. The sensor property is based on the domain-wall motion in magnetic strip in a spin-valve structure. The device temperature operating range can be configured by varying domain-wall thickness through the tuning magnetic material properties. TMR stack or magnetic stack with high GMR can improve the linearity or the resolution of the sensor.

Superconductivity

Sebastiano Peotta and Massimiliano Di Ventra [30] had an attempt to realize a superconducting memristor based on the phase-dependent conductance present in Josephson junctions. Superconducting memristor may have switching time in few picoseconds and this makes them extremely fast. A unique feature of this superconducting memristor is that it does not violate the Landauer bound on the minimum energy cost for reversible 
computation which do happen in case of irreversible computation with an ideal memristor and with other superconducting circuits. Superconducting memristors offer new venues for neuromorphic computation since they can be readily integrated with existing circuits with extremely fast operating frequencies.

Miscellaneous

Yuriy V. Pershin and Massimiliano Di Ventra [31] have shown how a network of memristors solves mazes in a massively-parallel way by way of implementation on a computer. They claim that hardware implementation of the memristive processor is superior to any existing maze solving methods and hence will be the best choice when the complexity of the maze increases. Further, it is assumed that the memristive processor can facilitate the solution of many other computational problems for example the traveling salesman problem, graph theory problems, etc. The memristive processor have the potential that they can then be used as a complete computational device, or as a supplemental tool for traditional computing hardware. Thus memristors are expected to affect the basic sciences and technology in a diverse manner.

\section{B. MEMCAPACITOR AND MEMCAPACITIVE SYSTEMS}

Latest experimental finding of a nanoscale memristor [4] (fig. 11) has led to numerous investigations in the area of materials and systems that show memory in their current-voltage characteristics [32-36]. This has also initiated the theoretical investigation of memcapacitors and meminductors, namely, capacitors and inductors with memory, and their capacitance and inductance, respectively, depends on the past (internal states variable) through which the system has evolved [37].

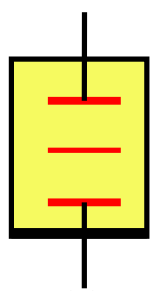

(a)

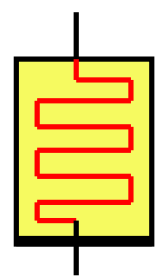

(b)

Fig. 11. Symbols of the two different memory device element: (a) memcapacitor, (b) memristor

\section{B.1. Memcapacitor: Definition and Properties}

Voltage-controlled memcapacitive system can be defined as

$q(t)=C\left(x, V_{C}, t\right) V_{C}(t)$

$\dot{x}=f\left(x, V_{C}, t\right)$

where $\mathrm{q}(\mathrm{t})$ is the charge on the capacitor at time $\mathrm{t}, \mathrm{V}_{\mathrm{C}}(\mathrm{t})$ is the corresponding voltage, and $\mathrm{C}$ is the memcapacitance (memory capacitance) which depends on some internal state vector of the system. Similarly, we can define an nth-order charge controlled memcapacitive system from the equations

$\mathrm{V}_{\mathrm{C}}(\mathrm{t})=\mathrm{C}^{-1}(\mathrm{x}, \mathrm{q}, \mathrm{t}) \mathrm{q}(\mathrm{t})$

$\dot{x}=\mathrm{f}(\mathrm{x}, \mathrm{q}, \mathrm{t})$

where $\mathrm{C}^{-1}$ is an inverse memcapacitance.

Properties

Charge is zero whenever the voltage is zero (equation 27). However, $I=0$ does not imply $q=0$, and thus the device has energy storage capacity and hence energy can be added to or removed from a memcapacitive system.

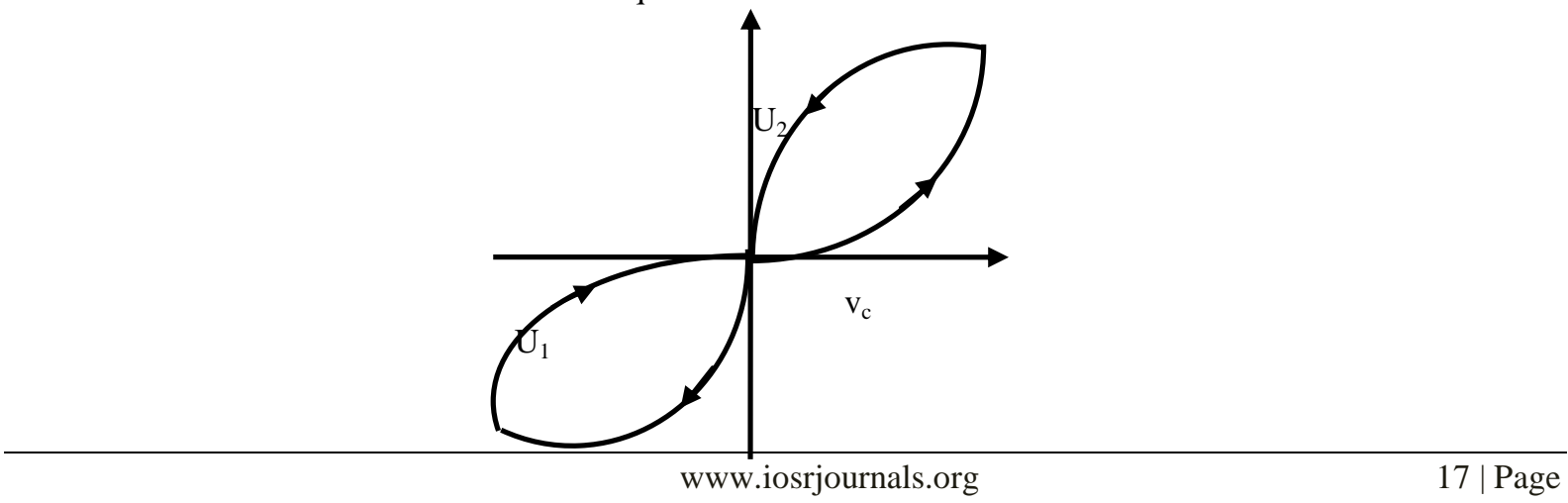


Fig. 12:Schematic of a pinched hysteresis loop of a memcapacitive system. The energy added to/removed from the system is the area between the curve and the $q$ axis. The areas of regions represented by $U_{1}$ and $U_{2}$ give the amount of energy added/removed in each half-period.

The area enclosed by the loops gives the energy, $U_{i}$, associated with some internal degree of freedom, added to/removed from the system (fig.12). Passive, dissipative and active systems are characterized by the sum $\mathrm{U}_{1}+$ $\mathrm{U}_{2}=,>$, $<0$. For a periodic input voltage, $\mathrm{V}_{\mathrm{C}}(\mathrm{t})=\mathrm{V}_{0} \cos (2 \pi \omega \mathrm{t})$, the $\mathrm{q}-\mathrm{V}_{\mathrm{C}}$ curve is a simple loop passing through the origin such that for a voltage-controlled device there are two values of the charge $q$ for a given voltage $\mathrm{V}_{\mathrm{C}}$, ( Fig. 12), and two values of the voltage $\mathrm{V}_{\mathrm{C}}$ for a given charge $\mathrm{q}$, in case of a charge-controlled system. The loop is anti-symmetric with respect to the origin. Like the case of memristive systems, a memcapacitive system behaves as a linear capacitor in the limit of infinite frequency, and as a non-linear capacitor in the limit of zero frequency, for if there exists a steady-state solution of Equations (27) and (28). The origin of this behavior rests again on the system's ability to adjust to a slow change in the control parameter (for low frequencies) and the reverse: its inability to respond to extremely high frequency oscillations.

Various systems are known to exhibit memcapacitive behavior including vanadium dioxide metamaterials, [33 ] nanoscale capacitors with interface traps or embedded nanocrystals, [38-41] and elastic capacitors [42,43]. Here we review metamaterial memcapacitor, nanopore memcapacitor and elastic membrane memcapacitor system.

\section{B 2. Memcapacitive systems}

B 2.1. Metamaterial memcapacitor

J. Martinez-Rincon, M. Di Ventra, and Yu. V. Pershin [44] have suggested and analyzed a solid state memcapacitor made of a multilayer structure embedded in a regular parallel plate capacitor (fig. 13).

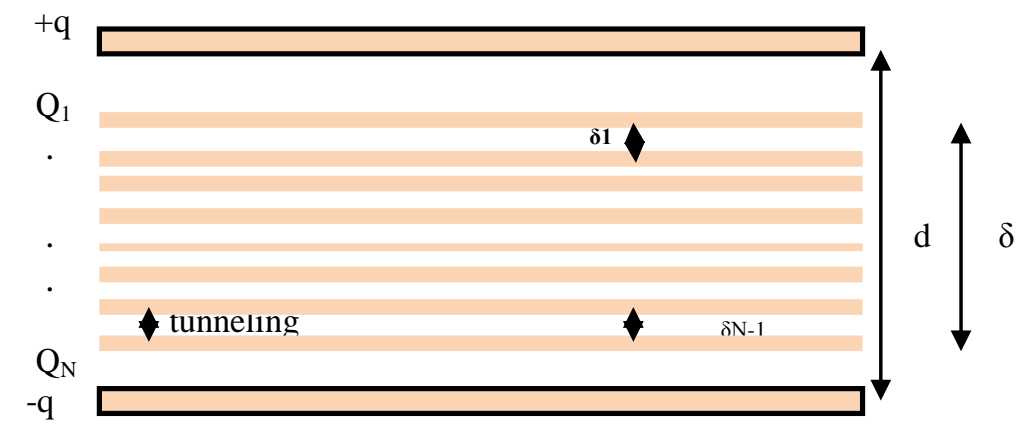

Fig. 13. General scheme of a solid-state memcapacitor. A metamaterial medium consisting of $N$ metal layers embedded into an insulator is inserted between the plates of a "regular" capacitor. It is assumed that the electron transfer between external plates of the capacitor and internal metal layers is negligible.

They have considered a metamaterial medium consisting of $N$ metal layers embedded into an insulator inserted between the plates of a regular parallel plate capacitor. The internal charges $Q_{k}$ can only be redistributed between the internal layers creating a medium polarization.

The resulting system has features of frequency-dependent hysteresis, diverging, and negative capacitance arising due to the slow polarizability of the internal multilayer structure as a consequence of electron tunneling between the internal metal layers. Both the information stored in this memcapacitor in the analog form and its negative capacitance in a certain range of the external field, may find useful applications in electronics. In the structure shown in Fig.3, the external plate voltage is given by

$V_{C}=2 d E_{q}+\delta E_{1}+\left[\delta-2 \delta_{1}\right] E_{2}+\left[\delta-2\left(\delta_{1}+\delta_{2}\right)\right] E_{3}+\ldots+\left[\delta-2\left(\delta_{1}+\ldots \ldots \delta_{k-1}\right) E_{k} \ldots-\delta E_{N}\right.$

where $E_{q}=\mathrm{q} /\left(2 S \varepsilon_{0} \varepsilon_{\mathrm{r}}\right)$ is the electric field due to the charge $q$ at the external plate and $E_{k}=Q_{k} /\left(2 S \varepsilon_{0} \varepsilon_{\mathrm{r}}\right)$ is the electric field due to the charge $Q_{k}$ at the $k^{\text {th }}$ embedded metal layer.

The capacitance of the whole structure is 
$C=\frac{q}{V_{C}}=\frac{2 C_{0}}{2+\sum_{i=1}^{N}\left(\Delta-2 \Delta_{i-1}\right) \frac{Q_{i}}{q}}$

The electric field $E_{k, k+1}$ between two neighboring layers is obtained by adding the electric fields due to charges at all layers and at the external metal plates,

$$
\begin{aligned}
E_{k, k+1} & =-2 E q-E_{1}-E_{2^{-}} \ldots \ldots . . E_{k}+E_{k+1}+\ldots . .+E_{N} \\
& =\left\{-2 q-\left(Q_{1}+Q_{2}+\ldots .+Q_{K}\right)+\left(Q_{k+1}+\ldots \ldots .+Q_{N}\right)\right\} / 2 \varepsilon_{o} \varepsilon_{r} S
\end{aligned}
$$

The dynamics of the charge at a metal layer $k$ is then determined by the currents flowing to and from that layer,

$d Q / d t=I_{k-1, k}-I_{k, k+1}$

where $I_{k, k+1}$ is the tunneling electron current flowing from layer $k$ to layer $k+1$.

Fig. 14 represents the equivalent model of two layer memcapacitor.

(a)

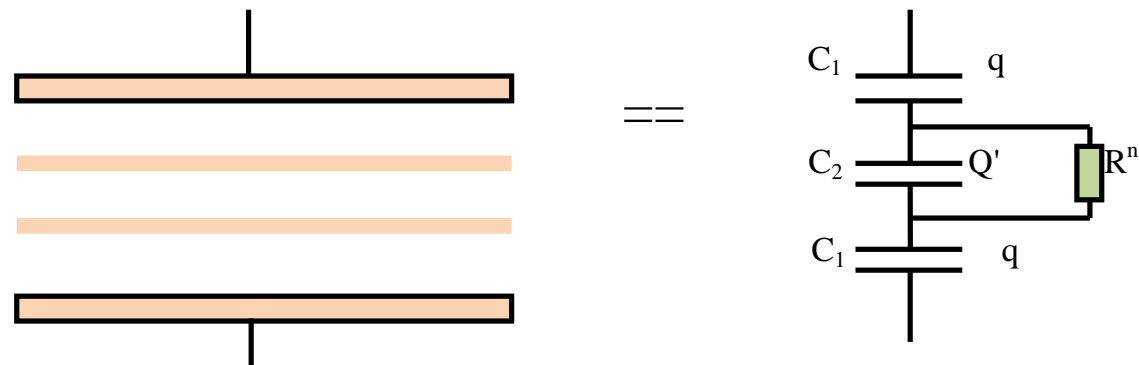

(b)

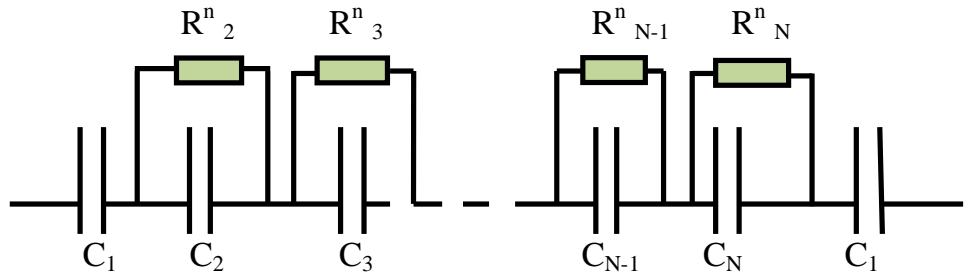

Fig. 14. (a) Equivalent circuit model of two-layer memcapacitor. Here, $C_{1}$ and $C_{2}$ are usual capacitors and $R^{n}$ is a nonlinear resistor for symmetrically embedded internal layers . (b) Equivalent circuit model of $N$-layer memcapacitor.

The capacitances $\mathrm{C}_{1}$ and $\mathrm{C}_{2}$ are

$C_{1}=2 C_{0} / 1-\Delta$ and $C_{2}=C_{0} / \Delta$

Equations describing the equivalent model are

$V_{C}=2 q / C_{1}+Q^{\prime} / C_{2}$

$d q / d t=d Q^{\prime} / d t+I_{R}$

whence the total capacitance $\mathrm{C}$ becomes

$C=q / V_{C}=C_{o} / 1+\Delta\left(Q^{\prime}-q\right) / q$

$d / d t\left\{\left(Q^{\prime}-q\right)\right\}=-I_{R}$

Simulation results [44] of two-layer memcapacitor with symmetrically positioned internal layers is shown in fig. 15. The parameter values used for simulation being $V_{0}=7.5 \mathrm{~V}, f=10 \mathrm{kHz}, d=100 \mathrm{~nm}, \delta=66.6 \mathrm{~nm}, S=10^{-4} \mathrm{~m}^{2}$, $\varepsilon_{\mathrm{r}}=5, U=0.33 \mathrm{eV}$, and $R=1 \Omega$. 

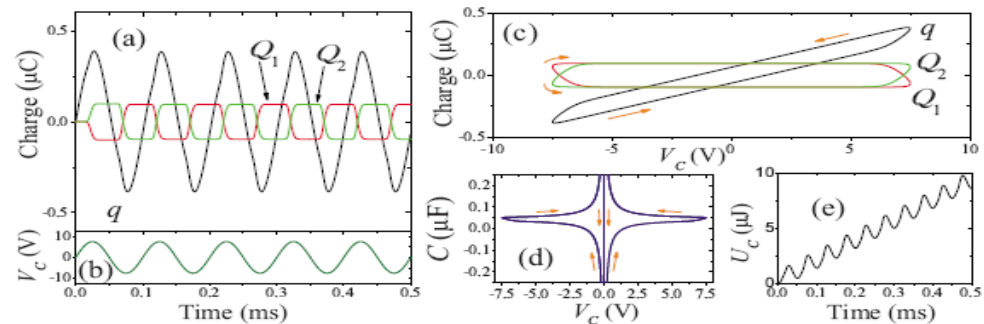

Fig. 15. Simulation results [19] of two-layer memcapacitor with symmetrically positioned internal layers. (a) The charge on internal metallic layers and memcapacitor plates as a function of time $t$. (b) Voltage on memcapacitor, $V_{C}$, as a function of time $t$.(c) Charge-voltage and (d) capacitance-voltage plots. (e) Added/removed energy as a function of time $t$. The parameter values used are $V_{0}=7.5 \mathrm{~V}, f=10 \mathrm{kHz}, d=100 \mathrm{~nm}, \delta=66.6 \mathrm{~nm}, S=10^{-4} \mathrm{~m}^{2}, \varepsilon_{\mathrm{r}}=5, U=0.33 \mathrm{eV}$, and $R=1 \Omega$.

\section{B 2.2. Nanopore memcapacitor}

A nanopore is an aperture of nanoscale dimensions across an insulating membrane.

Matt Krems,, Yuriy V. Pershin, and Massimiliano Di Ventra [46 ] have shown, using molecular dynamics simulations, that a nanopore sequencing setup acts as a memcapacitor, [45] The memcapacitive behaviour is due to two types of effects that happens at very different frequencies of the external bias. Schematic of the molecular dynamics geometry showing the buildup of charges of the opposite sign on each side of the nanopore due to a finite electric field $E$ is shown in fig. 16. The nanopore membrane is located at the center ( shown as parallel red lines). At high frequencies, memcapacitive effect is due to the finite mobility of ions in water and the slow polarizability of the ionic solution. At very low frequencies, memcapacitive effects occur as a result of the ion transport through the nanopore. These processes occur internally in the system and concern with time evolution of internal state variable.

When the system is subject to a periodic external electric field, electric field forces ions to accumulate at the two surfaces of the nanopore membrane, creating an effective capacitor.
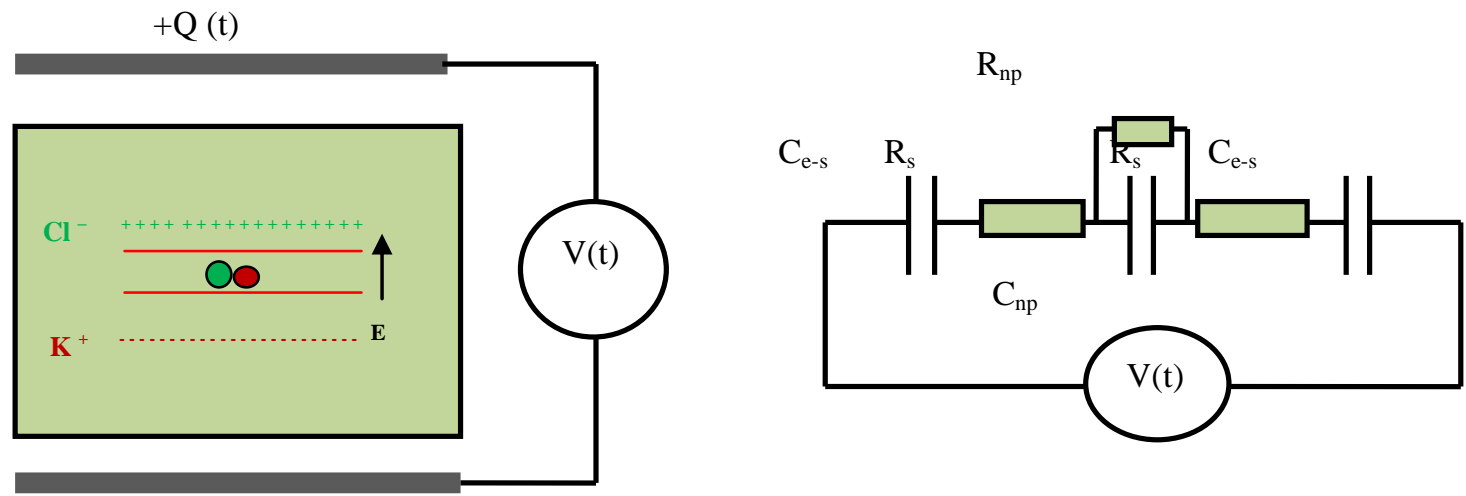

- Q (t)

Fig.16. (left) Schematic of the molecular dynamics geometry showing the buildup of charges of the opposite sign on each side of the nanopore due to a finite electric field $E$. The nanopore membrane is located at the center ( red lines). Top and bottom horizontal blue lines represent electrodes (holding plate charges $(Q(t))$ of the suggested experimental setup. (right) Simplified equivalent circuit model. After [46].

The equivalent circuit model is shown in fig. 16 . Here, $C_{\mathrm{e}-\mathrm{s}}$ denotes the external electrode- solution capacitance, $R_{\mathrm{s}}$ is the resistance of the solution, $R_{\mathrm{ss}}(>>R \mathrm{~s})$ is the resistance of the ion current through the pore, and $C_{\mathrm{ss}}$ is the capacitance of the membrane. The total voltage drop is then given by

$2 \mathrm{Q} / \mathrm{C}_{\mathrm{e}-\mathrm{s}}+2 \mathrm{R}_{\mathrm{S}} \mathrm{dQ} / \mathrm{dt}+\mathrm{Q} / \mathrm{C}_{\mathrm{ss}}=\mathrm{V}(\mathrm{t})$

The equation for the charge then takes the form

$\mathrm{dQ} / \mathrm{dt}=\mathrm{V}(\mathrm{t}) / 2 \mathrm{R}_{\mathrm{S}}-\mathrm{Q} / 2 \mathrm{R}_{\mathrm{S}} \mathrm{C}_{\mathrm{SS}}$ 
The long-time limit solution for the charge and capacitance in terms of microscopic parameters becomes

$$
\begin{aligned}
& Q(t)^{t \rightarrow \infty}=\frac{2 A \mu e n E_{0} \sin (\omega t)}{\left(1+\frac{1}{\omega^{2} \tau^{2}}\right) \omega^{2} \tau}-\frac{2 A \mu e n E_{0} \cos (\omega t)}{\left(1+\frac{1}{\omega^{2} \tau^{2}}\right) \omega} \\
& C(t)^{t \rightarrow \infty}=\frac{2 A \mu e n}{\left(1+\frac{1}{\omega^{2} \tau^{2}}\right) \omega^{2} \tau d}-\frac{2 A \mu e n \cot (\omega t)}{\left(1+\frac{1}{\omega^{2} \tau^{2}}\right) \omega d}
\end{aligned}
$$

where $A$ is the area of the membrane surface, $\mu=7.12 \times 10^{-8} \mathrm{~m}^{2} \mathrm{~V}^{-1} \mathrm{~s}^{-1}$, [43] is the ion mobility, similar for both types of ions, $e$ the ion charge, $n$ the density of ions in the bulk, and $\tau$ the relaxation time. The factor 2 takes into account the conductivity of channels $\left(\mathrm{K}+\right.$ and $\left.\mathrm{Cl}^{-}\right)$.

The simulation result [46] for charge Vs. voltage and that for capacitance Vs. voltage follows below. The arrows indicate the direction the voltage is swept in time and the numbers show the order in which the trace is generated. The loop narrows down as the frequency increases in conformity with the theoretical predictions in [37].
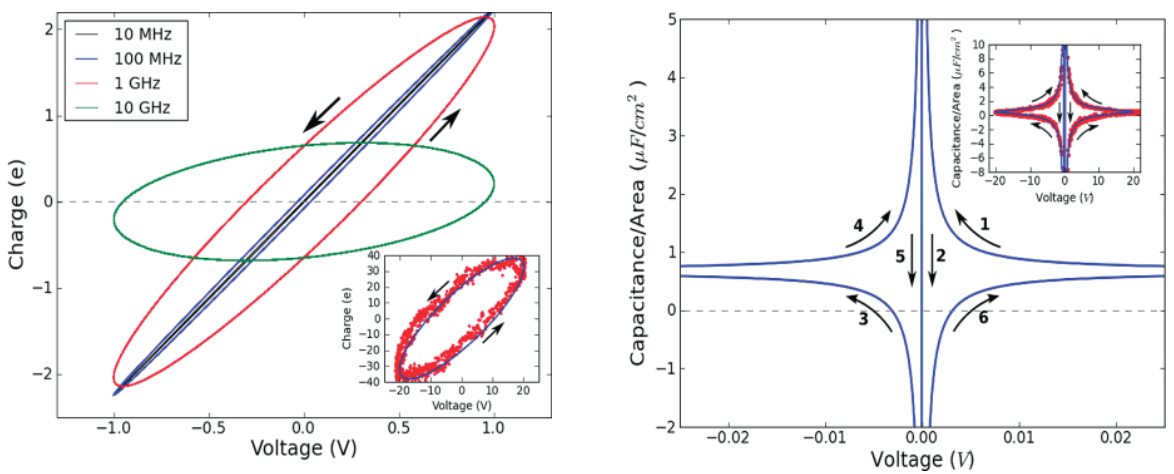

Fig.17. (left panel) Net charge $Q$ versus a periodic voltage of amplitude $V_{0}=1 \mathrm{~V}$ and different frequencies as obtained from equation (41). The inset shows the same quantity for $2 \mathrm{GHz}$ and $V_{0}=20 \mathrm{~V}$ compared with the charge obtained directly from. (right panel) Capacitance versus a periodic voltage of frequency $f=10 \mathrm{MHz}$ and amplitude $V_{0}=1 \mathrm{~V}$ as obtained from equation (42). In the inset we show the same quantity for $f=2 \mathrm{GHz}$ and $V_{0}=20 \mathrm{~V}$ compared with the capacitance obtained directly from simulations. It is seen that $C$ can be both negative and diverging as the voltage approaches zero. The arrows indicate the direction the voltage is swept in time and the numbers show the order in which the trace is generated. After [46].

This capacitor shows interesting features as a function of the frequency of the field, namely, a non-pinched hysteresis loop of the capacitance (memory-capacitance or memcapacitance) as a function of voltage which diverges at zero voltage and displays negative-capacitance properties (fig. 17).

\section{B 2.3. Elastic membrane memcapacitor}

The membrane memcapacitor (fig. 18) is a modification of a parallel-plate capacitor, in which one of the parallel plates is replaced by a strained membrane having two equilibrium positions (' 0 ' and ' 1 ') that can be used in memory applications.

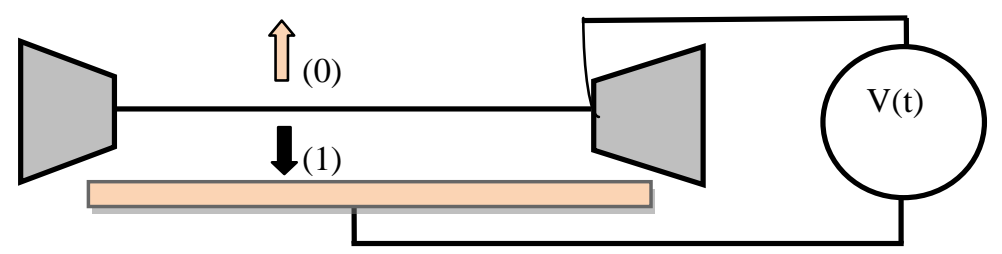

Fig. 18: Schematic of a bistable non-volatile elastic membrane memcapacitor connected to a voltage source V (t). The top plate of a regular parallel-plate capacitor is replaced by a flexible strained membrane. Because of two equilibrium positions of the membrane ( up/down arrow i.e. 0 and 1), stable high and low capacitance configurations are possible. After [47] 
If $m$ is the mass of the membrane and $\omega_{0}$ the natural angular frequency of the system then equation of motion for the membrane, typical of second-order voltage-controlled memcapacitor, is

$$
\begin{aligned}
\mathrm{q}(\mathrm{t}) & =\mathrm{C}_{\mathrm{o}} \mathrm{V}(\mathrm{t}) /\{1+\mathrm{y}(\mathrm{t})\} \\
& =\mathrm{C}(\mathrm{y}(\mathrm{t})) \mathrm{V}(\mathrm{t}) \\
\frac{d}{d \tau}\left[\begin{array}{c}
y \\
\dot{y}
\end{array}\right] & =\left[\begin{array}{l}
\dot{y} \\
\left.-4 \pi^{2} y\left(\frac{y^{2}}{y_{0}^{2}}-1\right)-\Gamma \dot{y}-\frac{\beta^{2}(\tau)}{(1+y)^{2}}\right]
\end{array}\right.
\end{aligned}
$$

where $\mathrm{C}_{\mathrm{o}}=\varepsilon_{0} \mathrm{~S} / \mathrm{d}, \mathrm{y}_{0}=\mathrm{z}_{0} / \mathrm{d}, \Gamma=2 \pi \gamma / \omega_{0}, \gamma$ is the damping constant, $\beta(t)=\left[2 \pi /\left(\omega_{0} d\right)\right] \sqrt{C_{0} /(2 m)} V(t)$. The time derivatives in Eq. (30) are taken with respect to the dimensionless time $\tau=\mathrm{t} \omega_{0} / 2 \pi$.

When the membrane memcapacitor is subjected to ac voltage, its dynamics can be periodic or chaotic depending on the frequency and amplitude of the ac voltage. We have shown below only the periodic case of [47]. A numerical solution of Equations $(43,44)$ with $\beta(t)=\beta_{0} \sin (\omega t)$ is plotted in fig. 19 for three different values of the applied voltage frequency. The hysteresis curves demonstrate typical behavior of memory elements at different range of frequencies [48]: non-linear dependencies at lower frequencies, pinched hysteresis loops at intermediate frequencies and linear behavior at higher frequencies.

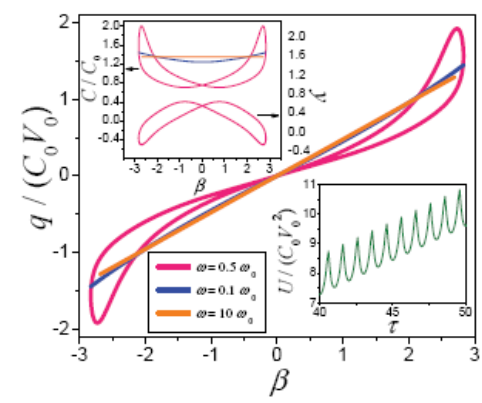

Fig. 19: $\mathrm{q}-\mathrm{V}$ and $\mathrm{C}-\mathrm{V}$ (top curves in the top inset) curves for a membrane memcapacitor calculated for a sinusoidal voltage input [47]. The bottom curve in the top inset represents $\mathrm{y}(\beta)$ at $\omega=0.5 \omega_{0}$. Hysteresis loops are seen at intermediate frequencies. The bottom inset is the energy dissipated by the system, given by $\mathrm{dU}=\mathrm{Vdq}$, calculated at $\omega=0.5 \omega_{0}$.

\section{Conclusion}

In this paper an attempt has been made to review the research and development in the field of memristive devices, including memristors and memcapacitors, and their various applications in analog electronic, spintronic and in the domain of neural network. In case of memristors, the study spans from the proposition on memristive devices by Leon O. Chua in 1971 to the first development of memristor in the laboratory by a group of HP scientists in the year 2008. Other examples are on thermistor, spintronic device, and a biological model on neural network. A separate section has been devoted to the various possible uses of memristor and memristor based circuits finding application in analog memory and computation, analog devices, associative memory, maze solver, temperature sensor and superconducting memristor. In case of memcapacitive systems, we have considered the most important memcapacitive system realizable from metamaterial (solid-state), nanopores (ionic transport through liquid) and elastic membrane (mechanical system), as the voltage and charge controlled devices. Before this we have discussed about memcapacitive systems and their properties. It is believed that these memristive and memcapacitive devices controlled by charge or voltage will revolutionalize future electronic memory devices with reference to the ease of operation, compactness, efficiency, cost and possibility of wider application domains.

\section{References}

[1] Di Ventra M, Electrical transport in nano scale systems (Cambridge University Press, Cambridge), $1^{\text {st }}$ Edn, 2008

[2] Pershin, Y V and Di Ventra M, Phys. Rev. B, 79 (15) (2009) 153307.

[3] Chua L O, IEEE Trans. Circuit Theory, CT-18 5 (1971) 507-519.

[4] Strukov D B, Snider G S, Stewart D R and Williams R S, Nature, 453 80-83 Tour J M and He T, Nature, 453 (2008) $42-43$

[5] Joglekar Y N and Wolf S J, Eur. J. Phys, 30 (2009) 661-675.

[6] Chua L O and Kang S M, Proc. IEEE, 642 (1976) 209-223.

[7] Sapoff M and Oppenheim R M, Proc. IEEE, 51 (1963) 1292-1305. 
[8]

Chen Y, Jung G Y, Ohlberg D A A et al, Nanotech., 14 (2003) 462-468.

Pershin Y V, and Di Ventra M, Phys. Rev. B, Condens. Matter, 78, (2008) 113309 1-4

Strukov D B, Snider G S, Stewart D R, and Williams R S, Nature (London), 453 (2008) 80-83.

Yang J J, Pickett M D, Li X et al, Nature Nanotechnology, 3 (2008) 429-433.

Driscoll T, Kim H T, Chae B G et al, arXiv:0901.0899 (2009).

Chua L O and Kang S M, Proc. IEEE, 64209 (1976).

Sapoff M, and Oppenheim R M, Proc. IEEE, 511292 (1963).

Pershin Yu V and Di Ventra M, Phys. Rev. B, 78 (2008) 113309.

Pershin Yu V and Di Ventra M, Phys. Rev. B, 77 (2008) 073301.

Wang X, Chen Y, Gu Y et al, Nature Neuroscience 3, 1165.

Hodgkin A L, and Huxley A F, Journal of Physiology, 117500 (2000).

Pershin Yuriy V and Di Ventra Massimiliano, arXiv:1011.3053v1.

Pershin Yuriy V and Di Ventra Massimiliano, arXiv: 0905.2935v3 (2010).

Zidana Mohammed Affan, Fahmyb Hossam Aly Hassan, Hussaina Muhammad Mustafa, Salamaa Khaled Nabil, Microelectronics Journal, DOI: 10.1016/j.mejo.2012.10.001 (2012).

Laiho Mika, Lehtonen Eero, 12th International Workshop on Cellular Nanoscale Networks and their Applications (CNNA), 978-1-42446678-8/10 @2010 IEEE (2010).

Qin Yu, Zhiguang Qin, Juebang Yu, Yuming Maol , International Conference on Communications, Circuits and Systems, DOI $: 10.1109 /$ ICCCAS.2009.5250356

Wang Wei, Yu Q, Yuhong Chunxiang Xu, 3 978-1-4244-4888-3/09 @2009 IEEE, (2009) 969-973.

Driscoll T, Quinn J, Klein S, et al, Applied Physics Letters, 97 (2010) 093502.

Li Zhi-Jun and Zeng Yi-Cheng, Chin. Phys. B, 224 (2013) 040502

Talukdar A, Radwan A G, Salama K N, Microelectronics Journal, 43 (2012) 169-175.

Mosad A G, Fouda M E, Khatib M A et al, Microelectronics Journal, 44 (2013) 814-820

Wang Xiaobin, Chen Yiran, Gu Ying, and Li Hai, IEEE Electron Device Letters, 311 (2010).

Peotta Sebastiano and Di Ventra Massimiliano, arXiv:1311.2975v1 (2013).

Pershin Yuriy V and Di Ventra Massimiliano, arXiv:1103.0021v2 (2011)

Pershin Y V, Fontaine S La and Di Ventra M, Phys. Rev. E, 80 (2009) 021926.

Driscoll T, Kim H T, Chae B G et al, Memory Metamaterials Science, 325 (2009) 1518

Driscoll T, Kim H T, Chae B G et al, Appl. Phys. Lett., 95 (2009) 043503.

Waser R and Aono M, Nat. Mater., 6 (2007) 833.

Scott J C and Bozano L D, Adv. Mater.,19 (2007) 1452.

Di Ventra M, Pershin Y V and Chua L O, Proc. IEEE, 97 (2009) 1717.

Kim Y, Park K H, Chung T Het al, Appl. Phys. Lett., 78 (2001) 934.

Fleetwood D M, Shaneyfelt M R, Warren W L, J et al, Microelectron. Reliab., 35 (1995) 403.

Su A Y K, Wang H L, Pilkuhn M H and Pei Z, Appl. Phys. Lett., 86 (2005) 062110.

Lee P F, Lu X B, Dai J Y et al, Nanotechnology, 17 (2006) 1202.

Nieminen H, Ermolov V, Nybergh, K et al, J. Micromech. Microeng., 12 (2002) 177.

artensky M B, arXiv:physics/0208048

R incon J Martinez, Di Ventra M, and Pershin Yu V, Physical Review B, 81 (2010) 195430

Di Ventra M, Pershin Y V, Chua L O, Proc. IEEE, 97 (2009) 1717-1724.

Krems Matt, Pershin Yuriy V and Di Ventra Massimiliano, Nano Lett., 10 (2010) 2674-2678.

Rincon Julian Martinez and Pershin Yuriy V, arXiv:1103.0910v1 (2011).

Di Ventra M, Pershin Y V and Chua L O, Proc. IEEE, 97 (2009) 1717. 